\title{
Esporas de Clostridium difficile y su relevancia en la persistencia y transmisión de la infección
}

\author{
Jonathan Barra-Carrasco, Cristian Hernández-Rocha, Patricio Ibáñez, Ana M. Guzmán-Durán, \\ Manuel Álvarez-Lobos y Daniel Paredes-Sabja
}

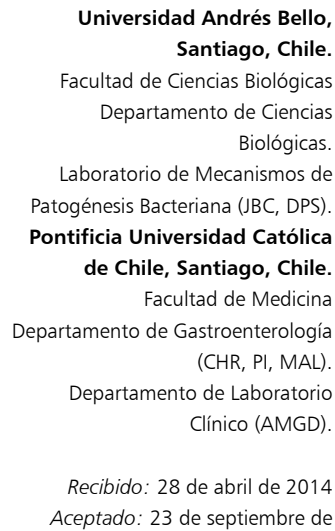

Universidad Andrés Bello Santiago, Chile.

Facultad de Ciencias Biológicas Departamento de Ciencias Biológicas

Laboratorio de Mecanismos de Patogénesis Bacteriana (JBC, DPS). Pontificia Universidad Católica

de Chile, Santiago, Chile. Facultad de Medicin

Departamento de Gastroenterología (CHR, PI, MAL).

Departamento de Laboratorio Clínico (AMGD)

Recibido: 28 de abril de 2014 Aceptado: 23 de septiembre de

Conflictos de interés: Los autores declaran no tener conflictos de interés

Financiamiento: Este trabajo fue financiado por Fondo Nacional de Ciencia y Tecnología de Chile (FONDECYT Regular 1110569), Fondos de la Vicerrectoría de Investigación de la Universidad Andrés Bello (DI-275-13/R) Fondo de Fomento al Desarrollo Científico y Tecnológico (FONDEF IdeA CA13/10077) (to D.P.-S); Fondos Nacional de Innovación en Salud CONICYT-FONIS SA1212197, FONDECYT Regular 1131012 y de Centro de Investigaciones Médicas de la Pontificia Universidad Católica de Chile (CIM-UC PG 06/13) M.A-L y FONDECYT de Inicio 11130502 de C.H-R

Correspondencia a: Daniel Paredes-Sabja

daniel.paredes.sabja@gmail.com

\section{Clostridium difficile spores and its relevance in the persistence and transmission of the infection}

C. difficile is an anaerobic spore former pathogen and the most important etiologic agent of nosocomial and community acquired antibiotics associated diarrheas. C. difficile infections (CDI) are responsible for an elevated rate of morbidity in developed and developing countries. Although the major virulence factors responsible for clinical symptoms of CDI are the two toxins TcdA and TcdB, C. difficile spores are the main vehicle of infection, persistence and transmission of CDI. Recent work has unrevealed unique properties of $C$. difficile spores that make them remarkable morphotypes of persistence and transmission in the host, including their resistance to antibiotics, the host immune response and disinfectants. The present review summarizes relevant aspects of $C$. difficile spore biology that have major implications from a clinical and medical perspective.

Key words: Clostridium difficile; spores; nosocomial diarrhea.

Palabras clave: Clostridium difficile, esporas, diarreas nosocomiales.

\section{Introducción}

\footnotetext{
C
} lostridium difficile es una bacteria grampositiva, anaerobia estricta y formadora de esporas. La infección asociada a $C$. difficile (IACD) es una de las principales infecciones asociadas a la atención de salud (IAAS) en el mundo ${ }^{1}$. El cuadro clínico de las IACD puede ir desde una diarrea leve y auto limitada hasta una colitis fulminante con pseudomembranas y megacolon tóxico ${ }^{2}$. La tasa de mortalidad de las IACD es aproximadamente de 5\%; sin embargo, uno de los mayores problemas de las IACD es su elevada tasa de recurrencia, la que puede alcanzar tasas de $20 \%$ después de un primer episodio ${ }^{3}$. Clostridium difficile secreta dos principales factores de virulencia, la enterotoxina TcdA y la citotoxina $\mathrm{TcdB}^{4}$. Ambas toxinas se unen a receptores celulares promoviendo su internalización a través de endosomas, causando la alteración del citoesqueleto de actina, apoptosis, disrupción de la barrera epitelial, y una masiva respuesta inflamatoria ${ }^{5}$. Otro factor de virulencia presente en algunos aislados clínicos es la toxina binaria CDT (Clostridium difficile transferasa), la cual se une al receptor de lipoproteína ${ }^{6}$ para luego ser translocada hacia el citosol y ADP-ribosilar la actina de forma irreversible promoviendo un re-ordenamiento del citoesqueleto de actina ${ }^{5}$. Recientemente, un estudio clínico sugiere que la toxina binaria podría estar involucrada en la recurrencia de las IACD ${ }^{8}$.

Durante la última década, el número de casos reportados de IACD ha experimentado un dramático incremento en la incidencia, con mayores tasas de mortalidad, recurrencia y de casos graves ${ }^{9-11}$. Estos cambios epidemiológicos se deberían, en parte, a la aparición de cepas epidémicas pertenecientes al ribotipo $027^{12,13}$. Se han descrito brotes de IACD causados por estas cepas con tasas de mortalidad y recurrencia cercanas a 15 y $30 \%$, respectivamente ${ }^{9-11}$. Otros estudios han sugerido un aumento de la gravedad de las IACD y de la frecuencia de colectomía ${ }^{14}$. La elevada virulencia de cepas pertenecientes al ribotipo 027 se debe en parte a que $C$. difficile posee un genoma muy plástico que le permite adaptarse a las presiones selectivas del entorno mediante la adquisición de nuevos genes, los que se transformarían en factores de virulencia y/o de adaptación al hospedero con potencial impacto en la gravedad de las IACD ${ }^{15}$. En esta línea, recientemente se ha reportado la aparición de cepas resistentes a fluoroquinolonas ${ }^{16}$, debido a una sustitución en Thr82Ile del gen gyrA. Otra mutación involucrada en la adquisición de una mayor virulencia del ribotipo 027 corresponde a una deleción en el gen que codifica un represor para la producción de toxina, $t c d C^{17}$ y que determinaría un incremento en la producción de toxinas con el consecuente aumento de citotoxicidad comparado con otros ribotipos ${ }^{18}$. Finalmente, nuevos ribotipos (i.e. $078,106,001,244)$ también han sido asociados a mayor incidencia de IACD graves ${ }^{19-21}$.

Clostridium difficile posee una serie de desventajas en su capacidad de colonizar el colon comparado con 
otros microorganismos como Bacteroides spp, otros Clostridium, Fusobacterium spp y Bifidiobacterium $\mathrm{spp}^{22}$. Por este motivo, normalmente no se encuentra formando parte de la microbiota colónica. Los tratamientos con antimicrobianos, sin embargo, provocan una alteración en esta microbiota, generando las condiciones para que esporas de $C$. difficile ingeridas inadvertidamente, germinen y se desarrollen hasta la forma vegetativa adulta de C. difficile y colonizen el colon ${ }^{23}$. Se desconoce cuál es la tasa infectante mínima de esporas requerida para producir una IACD. Clostridium difficile tiene la capacidad de generar esporas que son el vehículo de persistencia en el ambiente y en el colon ${ }^{24}$ donde son capaces de sobrevivir a los ataques del sistema inmune innato del hospedero ${ }^{25}$. Durante su ciclo infectante, $C$. difficile inicia un proceso de esporulación produciendo una elevada cantidad de esporas que son diseminadas al ambiente a través de las deposiciones ${ }^{26}$. La alteración de la microbiota favorece la germinación de esporas; posiblemente esté mediada por el incremento en los niveles de colatos y derivados ${ }^{27}$. Las esporas son eliminadas por las deposiciones al medio ambiente, donde pueden sobrevivir largo tiempo y probablemente representan la principal forma infectante de $C$. difficile, por lo que el manejo ambiental adecuado en los diferentes centros de atención de salud es crítico para evitar brotes ${ }^{28}$. Las esporas de $C$. difficile que no fueron eliminadas en las deposiciones pueden persistir en el tracto colónico del paciente, lo que pudiese favorecer los episodios de recurrencia de IACD. Los eventos de recurrencia de IACD se pueden clasificar como: recaida, si es causada por la misma cepa responsable del primer episodio, lo que representan $\sim 75 \%$ de las recurrencias; re-infección, si es causada por una cepa diferente a la del episodio inicial, con una frecuencia de $\sim 25 \%$ de todos las recurrencias ${ }^{3}$. En ambos casos, la recurrencia de IACD se vería favorecida por una modificación extrema de la microbiota colónica y la consecuente alteración de los mecanismos de defensa intrínsecos de esta microbiota contra de las infecciones por bacterias patógenas ${ }^{29,30}$.

A pesar de la enorme relevancia que implica el morfotipo de la espora en las IACD, los trabajos que consideran el estudio de las esporas de $C$. difficile dan cuenta sólo de $5,1 \%$ de todos los artículos científicos relacionados con C. difficile en PubMed (hasta el 20 de agosto de 2014). Por consiguiente, el objetivo de esta revisión es resumir el papel de las esporas de $C$. difficile en la persistencia y transmisión de las IACD, así como el conocimiento acerca de aspectos fundamentales sobre la formación, germinación y resistencia de sus esporas.

\section{Formación de la espora de C. difficile durante la IACD}

A diferencia de otras enfermedades entéricas causadas por enteropatógenos no formadores de esporas, C. diffi- cile, emplea una estrategia única y altamente sofisticada para persistir en el ambiente colónico del hospedero -la esporulación- la que se inicia cuando las condiciones ambientales son desfavorables para su sobrevivencia ${ }^{31}$ y culmina con la formación de una espora durmiente y altamente resistente a múltiples factores de estrés. El inicio del ciclo de esporulación comienza cuando quinasas histidinas huérfanas detectan estímulos ambientales y comienzan a fosforilar al regulador maestro de la esporulación, Spo0A ${ }^{31,32}$. El genoma secuenciado de C. difficile codifica cinco quinasas histidinas huérfanas (CD1352, CD1492, CD1579, CD1949 у CD2492) ${ }^{33}$. Estudios genéticos han demostrado que la inactivación de CD2492 reduce 3,5 veces la eficiencia de esporulación ${ }^{33}$. Al igual que en Bacillus subtilis, el circuito regulatorio del proceso de esporulación resulta en una cascada de activación de los factores sigma de la ARN polimerasa (i.e., $\sigma^{\mathrm{F}}, \sigma^{\mathrm{E}}, \sigma^{\mathrm{G}}$ y $\left.\sigma^{\mathrm{K}}\right)^{34}$ específicos de la esporulación. Si bien los cuatro factores sigma están presentes en los genomas que hasta la fecha han sido secuenciados de $C$. difficile, y los cambios morfológicos durante el desarrollo de la espora son similares a los de B. subtilis, la regulación temporal difiere significativamente de este segundo $^{35}$. Estudios transcriptómicos han demostrado que $C$. difficile comienza a esporular cuatro hs después de iniciada la infección en un modelo murino de IACD, observándose esporas maduras $14 \mathrm{~h}$ después de este inicio $^{36}$. Recientemente, Deakinet y cols. ${ }^{37}$, demostraron que cepas de $C$. difficile que no forman esporas, producto de una mutación en el regulador maestro de esporulación, spo0A, son incapaces de persistir y diseminarse en un modelo murino de IACD.

A pesar de que no existe evidencia directa de esporulación y persistencia de esporas de $C$. difficile en el tracto colónico de humanos, es posible que estos procesos sean similares a los reportados en modelos murinos ${ }^{36,37}$. Estos datos concuerdan con estudios clínicos donde $50 \%$ de los pacientes que se recuperan de un episodio de IACD se convierten en diseminadores asintomáticos de sus esporas por un período de 1-4 semanas después de la antibioterapia ${ }^{26,38}$. Un estudio reciente ha demostrado que las esporas de $C$. difficile son capaces de adherirse con una elevada eficiencia a células epiteliales in vitro, llegando a niveles de $60 \%$ de adherencia después de tres horas de infección ${ }^{24}$. Si bien la(s) proteína(s) de la espora y el(los) receptor(es) celular(es) que median la adherencia de esporas no han sido identificados, evidencia preliminar sugiere que es receptor-dependiente ${ }^{39}$. Debido a la importancia del proceso de esporulación para la persistencia de $C$. difficile en el hospedero, es esencial poder contar con estudios que permitan identificar las moléculas o señales que gatillan el proceso de esporulación con el fin de poder desarrollar análogos químicos que inhiban la esporulación in vivo y/o su persistencia en el hospedero. 


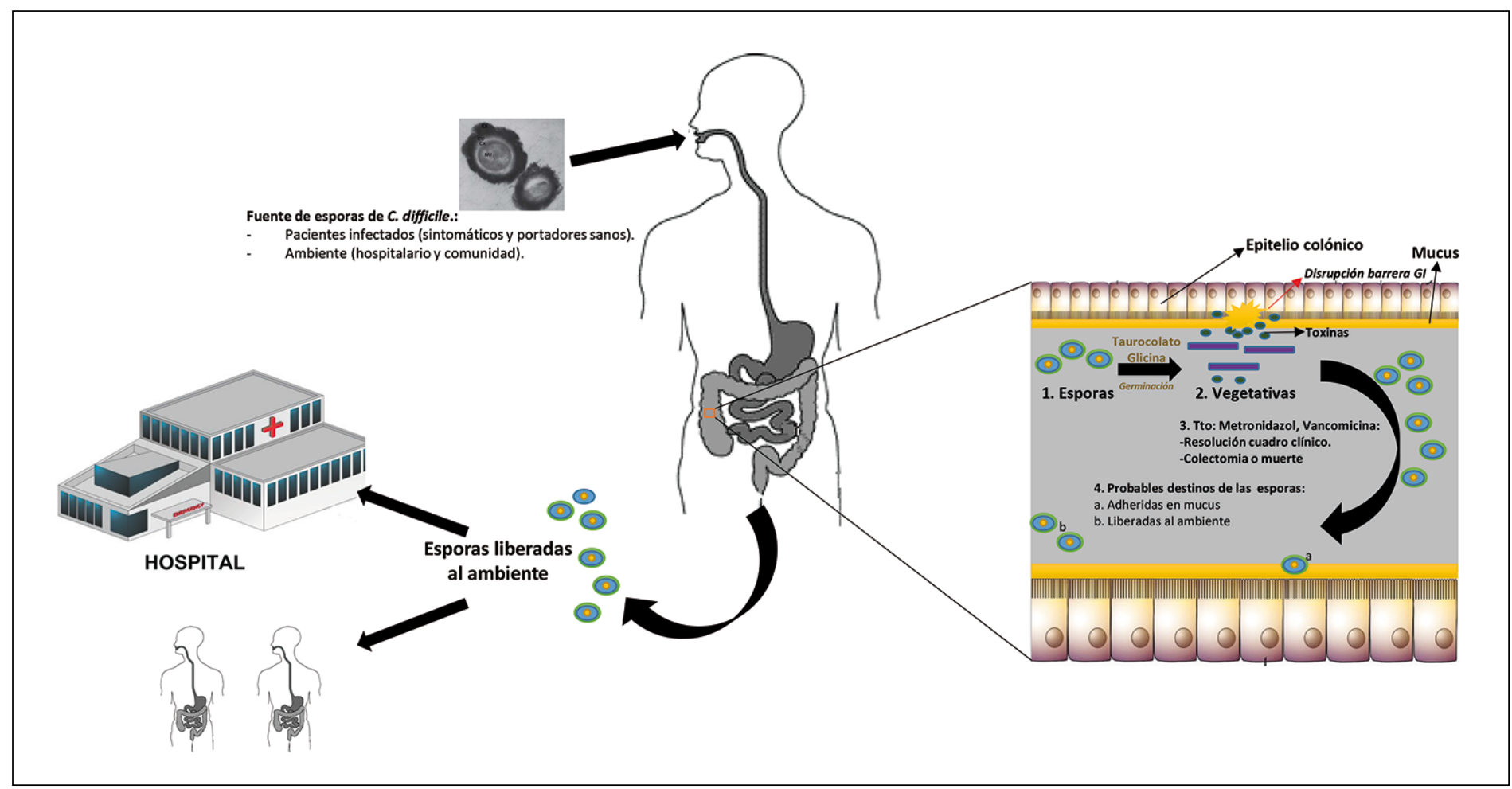

Figura 1. Esquematización del ciclo infectante de las IACD y de los mecanismos de infección, patogénesis, persistencia y diseminación de la IACD. El ciclo infectante de las IACD comienza con la ingestión de esporas por parte del paciente. Las esporas son capaces de resistir el ambiente gástrico ácido y el tránsito intestinal hacia el colon. Una vez en el colon, las esporas de C. difficile en presencia de factores favorables (i.e alteración de la microflora por tratamiento antimicrobiano, aumento de taurocolato y/o glicina), germinan y comienzan su ciclo vegetativo de crecimiento, produciendo toxinas las cuales provocan una fuerte respuesta inflamatoria por parte del hospedero y la disrupción de la mucosa colónica (i.e alteración del citoesqueleto de actina y apoptosis) provocando los síntomas de la IACD. Al mismo tiempo estas formas vegetativas de C. difficile comienzan un ciclo de esporulación generando esporas que ya sea se mantienen en al ambiento colónico (i.e., provocando cuadros de recurrencia) o son diseminadas al ambiente donde permanecen dormantes hasta infectar otro paciente donde comienza un nuevo ciclo infectante (Adaptada de ${ }^{23,28,39}$ ).

Esta inhibición de la esporulación ha sido ampliamente estudiada en levaduras y se han identificado fármacos catiónicos anfotéricos capaces de frenar este proceso ${ }^{40}$. La única molécula conocida a la fecha que inhibe la esporulación de $C$. difficile es el antibacteriano inhibidor de la ARN polimerasa, fidaxomicina, el que, a pesar que tiene el potencial de fracasar por resistencia durante el tratamiento y, por esta razón, se recomienda su uso sólo en casos específicos, ha demostrado disminuir la tasa de recurrencia comparado con otros antimicrobianos ${ }^{41}$. El mecanismo preciso de cómo afecta la esporulación no está del todo claro ${ }^{42}$. En suma, la inhibición del proceso de esporulación podría constituir un nuevo blanco terapéutico para combatir las IACD. Para mayores detalles del proceso de formación de esporas de $C$. difficile, se recomienda consultar la referencia 43.

\section{Esporas de C. difficile como vector de transmisión}

Las esporas de $C$. difficile son metabólicamente durmientes y pueden sobrevivir por largos períodos (i.e., años) en el ambiente, convirtiéndolas en un eficiente morfotipo de transmisión. Recientemente se demostró que la contaminación del ambiente hospitalario con esporas de $C$. difficile contribuye con $\sim 38 \%$ de los casos de IACD (Figura 1) en pacientes hospitalizados bajo terapia con antimicrobianos $^{44}$. Los focos que contribuyen a la diseminación de las esporas en el ambiente hospitalario son diversos. Estudios han demostrado que el personal médico puede actuar como vector de transmisión de esporas de $C$. difficile $e^{26,45,46}$. Recientemente, Landelle y cols. ${ }^{47}$, observaron que cerca de $25 \%$ del personal a cargo del cuidado de pacientes con IACD tenía sus manos contaminadas con esporas de $C$. difficile debido al no uso de guantes. Los pacientes con IACD son considerados diseminadores de esporas de $C$. difficile, a través de sus deposiciones, piel y ropa ${ }^{26,45,46}$. Debido a esto, es vital un diagnóstico temprano de los casos de IACD, lo que permite identificarlos oportunamente y aislarlos. Un adecuado aislamiento de cada caso, de preferencia en habitaciones individuales y el uso de guantes y delantales desechables por parte del personal de salud, permitirían evitar la diseminación de la 
infección ${ }^{28}$. Cabe mencionar que si bien, el uso de guantes protege del contacto directo con esporas de C. difficile, éstos deben ser autoclavados antes de su eliminación ${ }^{48}$. Por otra parte, si las salas de pacientes con IACD no son adecuadamente desinfectadas, se convierten en reservorios de $C$. difficile ${ }^{49}$. Interesantemente, la diseminación aérea de esporas de $C$. difficile podría ocurrir durante el aseo de las camas de los pacientes infectados contaminando paredes y ductos de ventilación ${ }^{50-54}$, alcanzando recuentos de 53-476 ufc/ $/ \mathrm{m}^{35}$. A pesar de la información disponible, se requieren más estudios para evaluar cómo contribuye de forma cuantitativa cada uno de los factores ambientales a la transmisión de las IACD. En resumen, las esporas de $C$. difficile pueden persistir fácilmente en el ambiente hospitalario, por lo que su erradicación del ambiente hospitalario podría ayudar a reducir las tasas de infección intrahospitalarias.

\section{Factores de resistencia en la espora de C. difficile}

Las esporas bacterianas poseen factores bastante conservados involucrados en la resistencia hacia las noxas ambientales $^{56}$. El núcleo de las esporas posee un bajo contenido de agua ( 25 a $50 \%$ en peso húmedo), niveles elevados de ácido dipicolínico (DPA, 25\% del peso seco del núcleo) quelado con calcio (Ca-DPA) en una razón 1:1 y un ADN saturado con proteínas $\alpha / \beta$-SASP (proteínas ácidas pequeñas y solubles), que contribuyen a las propiedades de resistencia de las esporas. El núcleo está rodeado de una membrana significativamente comprimida, en la cual los fosfolípidos están inmóviles ${ }^{57}$, contribuyendo a una baja permeabilidad, incluso hacia el agua ${ }^{58}$. La membrana interna está rodeada por una pared de peptidoglicano, llamada pared germinal, que se transforma en la pared celular de células en crecimiento una vez terminada la germinación; esta capa no juega rol alguno en la resistencia de esporas. Sobre la pared germinal se encuentra una corteza de peptidoglicano con tres modificaciones estructurales importantes: solamente un cuarto de los residuos de ácido $N$-acetilmurámico (NAM) están sustituidos con péptidos cortos, otorgándole a la corteza un menor grado de entrecruzamiento y por consiguiente, mayor flexibilidad que la pared germinal; un cuarto de los residuos NAM lleva un residuo simple de L-alanina, una modificación no encontrada en la pared germinal. Aproximadamente cada segundo residuo de ácido murámico en la corteza de peptidoglicano ha sido convertido a murámico- $\delta$-lactamo (MAL), una modificación que no se encuentra en la pared germinal ${ }^{59,60}$. El residuo MAL actuaría como elemento de reconocimiento para las enzimas hidrolíticas de la corteza ${ }^{61}$. Rodeando la corteza está la membrana externa, que si bien juega un rol esencial durante el ensamblaje de la espora, no juega un rol en las propiedades de resistencia de las esporas. Por sobre la membrana externa hay una cubierta proteácea, formada por más de 70 proteínas en $B$. subtilis, que otorga resistencia hacia enzimas hidrolíticas de peptidoglicano y proteasas cuyos pesos moleculares sean mayores a $14 \mathrm{kDa}^{62-65}$. Algunas especies como $C$. difficile, poseen una capa más externa llamada exosporium, que presenta proyecciones de filamentos que podrían jugar roles esenciales en la interacción espora-hospedero ${ }^{39,43,66} \mathrm{y} / \mathrm{o}$ en la resistencia de las esporas. Los primeros estudios en factores de resistencia de esporas de $C$. difficile han identificado dos factores con un rol en la resistencia; estos son: $\mathrm{CdeC}$, el que está involucrado en la resistencia de la espora a etanol, calor y lisozima ${ }^{65}$ y CotA, involucrada en la pérdida de la resistencia al calor en un sub-grupo de la población total ${ }^{67}$. Ambos factores alteran el proceso de ensamblaje de las capas externas de las esporas de $C$. difficile. Sin embargo, no existen estudios moleculares que hayan caracterizado el rol de factores clásicos de resistencia de esporas bacterianas en $C$. difficile, como aquellos relacionados con deshidratación del núcleo de la espora (las proteínas SpmAB, DacB y SpoVA) ${ }^{68,69}$ y protección del ADN como las proteínas $\alpha / \beta$-SASP y $\mathrm{Ca}-\mathrm{DPA}^{56}$. En este contexto, son necesarios estudios en mecanismos de resistencia de esporas de $C$. difficile para desarrollar nuevas y eficientes estrategias que permitan eliminar las esporas tanto del hospedero como del ambiente hospitalario.

\section{Factores de persistencia de las esporas de $C$. difficile en el hospedero}

Durante el curso clínico de la IACD se ha observado un importante aumento de la cantidad de esporas excretadas por las deposiciones ${ }^{2,70,71}$, indicando que $C$. difficile esporula dentro del tracto intestinal, contribuyendo a la persistencia dentro del hospedero (Figura 2). La resistencia de las esporas de $C$. difficile a los antimicrobianos es tal, que éstas pueden persistir después de un curso terapéutico de metronidazol y vancomicina ${ }^{72}$. Se ha postulado que la presencia de diverticulosis en el colon podría actuar como un potencial reservorio de esporas de C. diffiicle, contribuyendo a la persistencia de las IACD; sin embargo, estudios recientes no avalan esta hipótesis ${ }^{73}$. Se ha estudiado los mecanismos de adherencia de células vegetativas de $C$. difficile ${ }^{74,75}$; no obstante, pocos estudios han evaluado la capacidad de las esporas de adherirse al epitelio intestinal. Recientemente Paredes- Sabja y cols. ${ }^{24}$, demostraron que las esporas de $C$. difficile se adhieren a células epiteliales intestinales Caco- 2 in vitro alcanzando niveles de adherencia de 60 a $70 \%$ del total de esporas, sugiriendo que la adherencia de esporas de $C$. difficile a la mucosa intestinal podría ser un posible mecanismo de persistencia en el hospedero. Otro estudio efectuado por nuestro grupo ${ }^{25}$ ha demostrado que las esporas de C. difficile, a pesar de ser eficientemente reconocidas e 


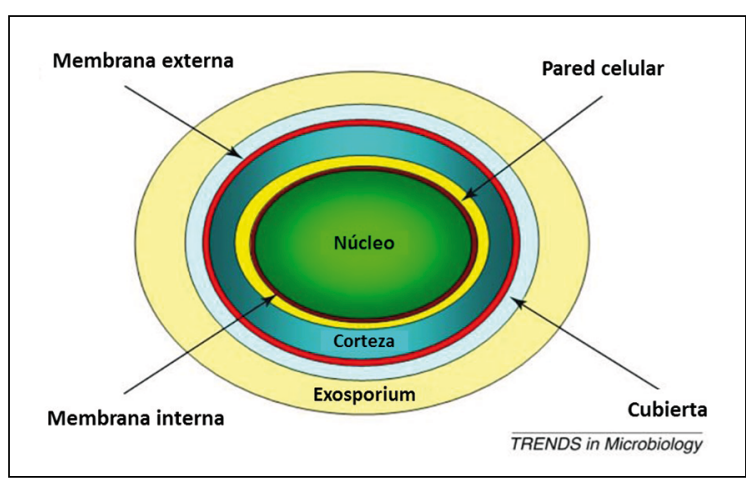

Figura 2. Esquematización de la estructura de una espora bacteriana. La capa más externa en algunas especies es el exosporium y está compuesta por $\sim 20$ proteínas. La capa inferior, llamada cubierta, está compuesta por $\sim 70$ proteínas en Bacillus subtilis y entrega protección contra agentes oxidantes y enzímas. Debajo de la cubierta está la membrana externa, la cual si bien es esencial durante la formación de la espora, con el tiempo se degrada y no juega rol alguno en la resistencia de esporas. Debajo de la membrana externa se encuentra la corteza de peptidoglicano, formado por un peptidoglicano específico para esporas con una estructura diferente al que se encuentra en la pared celular de células bacterianas. La corteza juega un rol fundamental en mantener el núcleo deshidratado actuando como una camisa de fuerza que mantiene al núcleo comprimido. Una vez degradada la corteza, esta presión se pierde con la consecuente hidratación del núcleo. Debajo de la corteza se encuentra la pared celular y está compuesta por peptidoglicano que pasará a formar parte de la pared celular de la célula naciente. Por debajo de la pared celular está la membrana interna. Debido a la fuerte presión osmótica del núcleo y la resistencia de la corteza, los fosfolípidos de de la membrana interna son inmóbiles y es impermeable a la difusión de solutos pequeños incluyendo agua. En el núcleo se encuentra el material genético y la maquinaria necesaria para re-iniciar el metabolismo. El núcleo se encuentra deshidratado y saturado de ácido dipicolínico quelado con calcio, el cual actúa como un protector hacia químicos y calor ${ }^{58}$. Modificado de (Paredes-Sabja, Setlow et al., 2011) con permiso de Elsevier.

internalizadas por células fagocíticas, permanecen latentes y sobreviven a los ataques de la maquinaria antibacteriana de los macrófagos, causando efectos citotóxicos en éstos. Ambos estudios sugieren que las esporas de $C$. difficile son capaces de adherirse a la mucosa intestinal y sobrevivir el ataque de células fagocíticas.

En cuanto a factores del hospedero, estudios de pacientes con IACD y en modelos animales, han demostrado que una microbiota colónica normal puede disminuir la producción y diseminación de esporas $y$, por consiguiente, de la persistencia de una IACD a través de mecanismos aún no identificados ${ }^{76}$.

\section{Desinfectantes y resistencia de las esporas de $C$. difficile en el ambiente}

El principal problema para erradicar las esporas de $C$. difficile del ambiente hospitalario es que, al igual que las esporas de otras especies bacterianas, éstas son resistentes a la mayoría de los desinfectantes utilizados en el ambiente hospitalario ${ }^{77}$. Algunos estudios han caracterizado la sobrevivencia de esporas de $C$. difficile a varios desinfectantes usados de rutina en la desinfección hospitalaria ${ }^{77-79}$. Por ejemplo, desinfectantes a base de etanol, no afectan la viabilidad de las esporas de C. difficile ${ }^{78}$. Compuestos de amonio cuaternario tampoco poseen actividad esporicida $^{77}$. Entre los desinfectantes alternativos con actividad esporicida se encuentran el hipoclorito de sodio, que a concentraciones de $5.000 \mathrm{ppm}$ reduce en siete ciclos logarítmicos la viabilidad de las esporas de $C$. difficile después de seis minutos de incubación a temperatura ambiente $^{78}$. Los nitritos acidificados y el glutaraldehído han demostrado una reducción intermedia en la viabilidad de las esporas, con reducciones de 4 a 7 ciclos logarítmicos después de 15 y 30 min de exposición ${ }^{80}$. Sin embargo, por ser sus efectos nocivos, estos desinfectantes no son recomendados ${ }^{81}$. Alternativamente, iones paracetilos y peróxido de hidrogeno vaporizado son una opción como agentes de desinfección, reduciendo la presencia de $C$. difficile entre 0,3 y 0,6 ciclos logarítmicos después de un minuto ${ }^{82,83}$. En resumen, el acotado repertorio de desinfectantes disponibles para eliminar esporas de $C$. difficile del ambiente hospitalario resalta la necesidad de desarrollar nuevos compuestos que eliminen de manera efectiva las esporas de $C$. difficile.

\section{Resistencia de esporas de C. difficile a antimicrobianos}

Las esporas de $C$. difficile son resistentes a todos los antimicrobianos conocidos, principalmente debido a que son metabólicamente inactivos y a que la membrana interna es impermeable a la difusión de moléculas pequeñas, lo que impide el ingreso de antimicrobianos hacia el núcleo de la espora $^{84-87}$. Los principales antibacterianos utilizados en el tratamiento de las IACD son metronidazol, vancomicina $\mathrm{y}$ recientemente fidaxomicina ${ }^{88}$. Estudios in vitro han demostrado que, tanto metronidazol como vancomicina, no inhiben la formación de esporas de C. difficile ${ }^{84,85}$, lo que es consistente con las elevadas tasas de recurrencia de IACD observadas en estudios clínicos ${ }^{89}$. En contraste, fidaxomicina, a concentraciones sub-inhibitorias, inhibe la formación de esporas de C. difficile in vitro ${ }^{42}$, y los estudios clínicos han demostrado que reduce a la mitad los episodios de recurrencia de IACD, comparado con vancomicina $^{41,90}$, debido posiblemente a la inhibición de la formación de esporas durante el tratamiento. Ninguno de estos tres antibacterianos afecta la viabilidad de las esporas latentes de $C$. difficile. Solamente vancomicina y fidaxomicina logran disminuir la eficiencia con la cual las esporas de C. difficile forman colonias (i.e., eficiencia de germinación) $)^{91}$. 


\section{Reactivación de las esporas de C. difficile: germinantes y germinación}

Las esporas bacterianas son capaces de retornar a su forma vegetativa en tan sólo cinco minutos después de entrar en contacto con los nutrientes, denominados germinantes. Estos germinantes son especie-específicos y captados por receptores de germinación pertenecientes a la familia GerA localizados en la membrana interna de la espora ${ }^{58}$. A diferencia de otras especies de los géneros Bacillus y Clostridium, $C$. difficile no posee los receptores canónicos de germinación de la familia GerA $^{58}$; por el contrario, los germinantes (i.e., taurocolato y L-glicina) son detectados por la proteasa serina, CspC, cuya localización se presume entre la interface de la cubierta y la corteza de peptidoglicano ${ }^{92}$ (Figura 1). El mecanismo mediante el cual CspC activaría a CspB y la subsecuente activación de la hidrolasa SleC, la que va a degradar la corteza de peptidoglicano de la espora, es desconocido ${ }^{93}$. La degradación de la corteza de la espora permite un cambio en la fluidez de la membrana interna que activaría los transportadores de Ca-DPA, expulsando Ca-DPA desde el núcleo con la consecuente rehidratación y reiniciación del metabolismo. A medida que se incrementa la hidratación del núcleo, las $\alpha / \beta$-SASP pierden la habilidad de asociarse al $\mathrm{ADN}^{69}$ y se activa la proteasa de germinación, $\mathrm{GPR}^{94}$, encargada de la degradación de las proteínas $\alpha / \beta$-SASP. En esta etapa de la germinación, las esporas pierden todas sus propiedades de resistencia y son extremadamente susceptibles a ser inactivadas por desinfectantes ${ }^{95}$ o el sistema inmune innato $^{25}$. La capa más externa de la espora, el exosporium, pareciera jugar un rol en la mantención de la latencia de las esporas actuando como un represor de la germinación, inhibiendo la reactivación de las esporas de C. difficile $e^{96}$. La eficiencia de germinación (i.e., el número de unidades formadoras de colonia por partículas de esporas) de las esporas de aislados clínicos de $C$. difficile varía entre 0,1 y $25 \%$ del total de las esporas ${ }^{96,97}$, muy por debajo del 70 a $90 \%$ de eficiencia de germinación observados en esporas de otras especies como B. subtilis y Clostridium perfringens ${ }^{98}$. Estudios nuestros recientes, indican que esta capa de exosporium se pierde después de cuatro meses de almacenamiento a temperatura ambiente, incrementando la eficiencia de germinación de las esporas en hasta 100 veces $^{108}$. En resumen, a diferencia de la elevada eficiencia de germinación de esporas (i.e., 70 a 90\% del total de esporas) observadas en otras especies ${ }^{98}$, la baja eficiencia de este proceso en $C$. difficile podría permitir su persistencia por períodos prolongados de tiempo en el tracto colónico del hospedero. Sin embargo, la pérdida de los factores de resistencia durante la germinación, sugiere que el desarrollo de terapias que involucren la modulación de la germinación, podrían contribuir a reducir al persistencia de $C$. difficile en el hospedero.

\section{Esporulación y producción de toxinas}

La regulación de la síntesis de toxina es un determinante crítico en la patogenicidad de $C$. difficile. Muchos factores ambientales influencian la producción de toxina, como por ejemplo, la disponibilidad de nutrientes, el potencial redox y la temperatura del entorno. Al respecto, se han encontrado tres reguladores involucrados en la producción de toxina por parte de $C$. difficile: Ccpa, mediando la represión dependiente de glucosa; CodY y Spo0A; estos dos últimos controlando eventos previos y posteriores a la fase exponencial de crecimiento ${ }^{104}$. En C. perfringens, $\sigma^{\mathrm{K}}$ es producido en células en la fase pre-divisional y se encuentra involucrado en la producción de enterotoxina ${ }^{105}$. En cuanto a $C$. difficile, un estudio mostró que en relación a los roles de los distintos factores sigma involucrados en esporulación, $C$. difficile parece ser cercano al organismo modelo, B. subtilis, más que a otras especies del género Clostridium $^{106}$.

Dos estudios independientes reportaron que mientras la mutación de $\operatorname{SpoOA}$ reduce la producción de TcdA, la mutación de $\mathrm{SigH}$ (factor sigma que regula positivamente algunos genes requeridos para la esporulación) estimularía la producción de $\operatorname{Tcd}^{104,107}$. Sin embargo, el efecto de estas dos proteínas en la expresión de toxinas por parte de $C$. difficile es indirecta y sus mecanismos todavía no han sido dilucidados.

\section{Nuevas estrategias para eliminar las esporas de $C$. difficile}

Recientes estudios han desarrollado novedosas estrategias de desinfección que involucran dos etapas. Una primera fase de "activación y germinación" de las esporas de $C$. difficile, seguida de una segunda etapa de "inactivación" donde las esporas ya germinadas son inactivadas por desinfectantes comúnmente utilizados en las rutinas de aseo de hospitales ${ }^{95,99}$. Interesantemente, estos estudios determinaron que luego de cinco minutos de la exposición a agentes germinantes, la espora germina y que la exposición de $10^{4}-10^{5}$ esporas a agentes germinantes antes de la desinfección disminuye el tiempo en que se inactivan desde 45 a sólo 10 minutos $^{95,99}$. Estas estrategias también podrían ser utilizada para incrementar la susceptibilidad de las esporas de $C$. difficile al sistema inmune innato del hospedero. De hecho, $90 \%$ de las esporas germinadas son inactivadas por macrófagos, cifra que sólo llega a 10\% en el caso de esporas dormantes ${ }^{25}$. Sin embargo, se requiere un acabado conocimiento de la cinética de reactivación de las esporas de $C$. difficile y factores que afectan la dormancia de la espora. Por ejemplo, aunque el taurocolato y el co-germinante L-glicina son suficientes para iniciar la germinación ${ }^{100}$, la eficiencia de germinación de las esporas de $C$. difficile incrementa cuando éstas entran en contacto con células epiteliales ${ }^{97}$. También existe una elevada variabilidad en la especificidad de la germinación de esporas 
de $C$. difficile que debe ser considerada al momento de desarrollar estrategias de inhibición de germinación o de inactivación de dos pasos. Por ejemplo, utilizando esporas de aislados clínicos, se demostró que el inhibidor quenodeoxicolato sólo inhibe la germinación de 6 de 15 (40\%) aislados clínicos de C. difficile ${ }^{101}$, sugiriendo que podrían haber diferencias en la especificidad del receptor CspC entre aislados clínicos. Como se mencionó con anterioridad, la eficiencia de germinación de las esporas de $C$. difficile también podría ser una variable importante en el desarrollo de estas terapias ${ }^{65,96}$, puesto que la fracción de esporas que no germina, permanece durmiente persistiendo por largos períodos de tiempo con el riesgo de causar IACD recurrentes. Son necesarios estudios para incorporar eficientemente la reactivación de las esporas de $C$. difficile con taurocolato y L-glicina en protocolos de desinfección que permitan erradicar las esporas del ambiente hospitalario.

Otra estrategia de modulación de la germinación de las esporas es aquella que busca la inhibición de la germinación mediante el uso de inhibidores del proceso. Recientemente, Howerton y cols., utilizaron un análogo químico del taurocolato (CamSA) para inhibir la germinación de esporas de C. difficile y lograron atenuar la virulencia de C. difficile en un modelo murino ${ }^{102}$. Estos hallazgos resaltan el potencial uso de inhibidores de la germinación de esporas como una estrategia profiláctica en contra de las IACD. Además, se ha visto que el uso de unidades móviles automáticas de luz UV, reduce significativamente la carga de esporas de $C$. difficile en superficies contaminadas de habitaciones de pacientes con $\mathrm{IACD}^{103}$. Claramente, son necesarios mayores esfuerzos para determinar los factores que permiten activar o bloquear la germinación de las esporas de $C$. difficile, tanto en superficies inertes o in vivo, con el fin de desarrollar estrategias más eficientes que pudieran eliminar completamente las esporas de $C$. difficile del ambiente hospitalario y del hospedero.

\section{Conclusiones}

Durante el curso de las IACD, $C$. difficile inicia un rápido y eficiente ciclo de esporulación contribuyendo a su persistencia en el hospedero y a su transmisión. Las esporas de $C$. difficile poseen similitudes ultraestructurales y propiedades de resistencia similares a las de esporas de otras especies bacterianas, permitiendo su supervivencia por períodos largos de tiempo en el hospedero y ambiente. A diferencia de otras especies, $C$. difficile posee un meca- nismo único para gatillar la germinación de sus esporas, consistente en la utilización de sales biliares (taurocolato) y el co-germinante L-glicina. Esta elevada especificidad de germinación permite que las esporas de $C$. difficile sólo germinen en el tracto colónico del hospedero y permanezcan durmientes por largos períodos de tiempo en el ambiente.

Las rutinas de desinfección basadas en desinfectantes libres de cloro no logran inactivar las esporas, por lo que futuras maneras para eliminar esporas del ambiente hospitalario podrían incluir estrategias que contemplan un primer paso de germinación seguido por un paso de inactivación. Igualmente, la modulación in vivo de la germinación de las esporas de $C$. difficile podría inhibir el retorno de las esporas al crecimiento activo o desencadenar su germinación para ser luego inactivadas por algún tratamiento anti- $C$. difficile como metronidazol y/o vancomicina o por el sistema inmune del paciente, evitando la recurrencia de la IACD.

\section{Resumen}

Clostridium difficile es un patógeno anaerobio, formador de esporas y el agente etiológico más importante de las diarreas asociadas a antimicrobianos, tanto nosocomiales como adquiridas en la comunidad. Las infecciones asociadas a $C$. difficile poseen una elevada tasa de morbilidad en países desarrollados y en vías de desarrollo. Los dos factores de virulencia principales son TcdA y TcdB, toxinas que causan la remodelación del citoesqueleto lo cual desencadena los síntomas clínicos asociados a esta enfermedad infecciosa. A pesar que las esporas de $C$. difficile son el principal vehículo de infección, persistencia en el hospedero y de transmisión, pocos estudios se han enfocado sobre este clave aspecto. Es altamente probable que la espora juegue roles esenciales en los episodios de recurrencia y de transmisión horizontal de la infección por este microorganismo. Estudios recientes han revelado características únicas de las esporas de $C$. difficile que las hacen capaces de ser altamente transmisibles y persistir dentro del hospedero. Más aún, algunas de estas propiedades están relacionadas con la resistencia de sus esporas a los desinfectantes más comúnmente usados en los recintos hospitalarios. La presente revisión resume los conocimientos más relevantes en la biología de las esporas de $C$. difficile, con un énfasis en aquellos aspectos con implicancias clínicas, incluido el control de infecciones en el ambiente hospitalario. 


\section{Referencias bibliográficas}

1.- Rupnik M, Wilcox M H, Gerding D N. Clostridium difficile infection: new developments in epidemiology and pathogenesis. Nat Rev Microbiol 2009; 7 : 526-36.

2.- Hookman P, Barkin J S. Clostridium difficile associated infection, diarrhea and colitis. World J Gastroenterol 2009; 15: 1554-80.

3.- Oka K, Osaki T, Hanawa T, Kurata S, Okazaki M, Manzoku T, et al. Molecular and microbiological characterization of Clostridium difficile isolates from single, relapse, and reinfection cases. J Clin Microbiol 2012; 50: 915-21.

4.- Schirmer J, Aktories K. Large clostridial cytotoxins: cellular biology of Rho/Rasglucosylating toxins. Biochim Biophys Acta 2004; 1673: 66-74.

5.- Davies A H, Roberts A K, Shone C C, Acharya K R. Super toxins from a super bug: structure and function of Clostridium difficile toxins. Biochem J 2011; 436: 517-26.

6.- Papatheodorou P, Carette J E, Bell G W, Schwan C, Guttenberg G, Brummelkamp T R, et al. Lipolysis-stimulated lipoprotein receptor (LSR) is the host receptor for the binary toxin Clostridium difficile transferase (CDT). Proc Natl Acad Sci USA 2011; 108: 16422-7.

7.- Schwan C, Stecher B, Tzivelekidis T, van Ham M, Rohde M, Hardt W D, et al. Clostridium difficile toxin CDT induces formation of microtubule-based protrusions and increases adherence of bacteria. PLoS Pathog 2009; 5:e1000626.

8.- Stewart D B, Berg A, Hegarty J. Predicting recurrence of $C$. difficile colitis using bacterial virulence factors: binary toxin is the key. J Gastrointest Surg 2013; 17: 118-24.

9.- Kuijper E J, Coignard B, Tull P. Emergence of Clostridium difficile-associated disease in North America and Europe. Clin Microbiol Infect 2006; 12 Suppl 6: 2-18.

10.- Kato H, Ito Y, van den Berg R J, Kuijper E J, Arakawa Y. First isolation of Clostridium difficile 027 in Japan. Euro Surveill 2007; 12:E070111 070113.

11.- Borgmann S, Kist M, Jakobiak T, Reil M, Scholz E, von Eichel-Streiber C, et al. Increased number of Clostridium difficile infections and prevalence of Clostridium difficile PCR ribotype 001 in southern Germany. Euro Surveill 2008; 13.

12.- Loo V G, Poirier L, Miller M A, Oughton M, Libman M D, Michaud S, et al. A predominantly clonal multi-institutional outbreak of Clostridium difficile-associated diarrhea with high morbidity and mortality. N Engl J Med 2005; 353: 2442-9.

13.- McDonald L C, Killgore G E, Thompson A, Owens R C Jr, Kazakova S V, Sambol S P, et al. An epidemic, toxin gene-variant strain of Clostridium difficile. N Engl J Med 2005; 353: 2433-41.

14.- Halabi W J, Nguyen V Q, Carmichael J C, Pigazzi A, Stamos M J, Mills S. Clostridium difficile colitis in the United States: a decade of trends, outcomes, risk factors for colectomy, and mortality after colectomy. J Am Coll Surg 2013; 217: 802-12.

15.- Stabler R A, Valiente E, Dawson L F, He M, Parkhill J, Wren B W. In-depth genetic analysis of Clostridium difficile PCR-ribotype 027 strains reveals high genome fluidity including point mutations and inversions. Gut Microbes 2010; 1: 269-76.

16.- He M, Miyajima F, Roberts P, Ellison L, Pickard DJ, Martin MJ, et al. Emergence and global spread of epidemic healthcare-associated Clostridium difficile. Nat Genet 2013; 45 : 109-13.

17.- Carter G P, Douce G R, Govind R, Howarth P M, Mackin K E, Spencer J, et al. The anti-sigma factor $\mathrm{TcdC}$ modulates hypervirulence in an epidemic BI/NAP1/027 clinical isolate of Clostridium difficile. PLoS Pathog 2011; 7: e1002317.

18.- Lanis J M, Heinlen L D, James J A, Ballard J D. Clostridium difficile 027/BI/NAP1 encodes a hypertoxic and antigenically variable form of TcdB. PLoS Pathog 2013; 9: e1003523.

19.- Huang H, Nord CE. Can metronidazole still be used for treatment of Clostridium difficile infections? Curr Infect Dis Rep 2009; 11: 3-6.

20.- Wilcox M H, Shetty N, Fawley W N, Shemko M, Coen P, Birtles A, et al. Changing epidemiology of Clostridium difficile infection following the introduction of a national ribotyping-based surveillance scheme in England. Clin Infect Dis 2012; 55: 1056-63.

21.- De Almeida M N, Heffernan H, Dervan A, Bakker S, Freeman J T, Bhally H, et al. Severe Clostridium difficile infection in New Zealand associated with an emerging strain, PCRribotype 244. N Z Med J 2013; 126: 9-14.

22.- Arumugam M, Raes J, Pelletier E, Le Paslier D, Yamada T, Mende D R, et al. Enterotypes of the human gut microbiome. Nature 2011; 473: 174-80.

23.- Sarker M R, Paredes-Sabja D. Molecular basis of early stages of Clostridium difficile infection: germination and colonization. Future Microbiol 2012; 7: 933.

24.- Paredes-Sabja D, Sarker M R. Adherence of Clostridium difficile spores to Caco-2 cells in culture. J Med Microbiol 2012; 61: 1208-18.

25.- Paredes-Sabja D, Cofre-Araneda G, Brito-Silva C, Pizarro-Guajardo M, Sarker M R. Clostridium difficile sporemacrophage interactions: spore survival. PLoS One 2012; 7: e43635.

26.- Sethi A K, Al-Nassir W N, Nerandzic M M, Bobulsky G S, Donskey C J. Persistence of skin contamination and environmental shedding of Clostridium difficile during and after treatment of $C$. difficile infection. Infect Control Hosp Epidemiol 2010; 31: 21-7.

27.- Theriot C M, Koenigsknecht M J, Carlson P E Jr, Hatton G E, Nelson A M, Li B, et al. Antibiotic-induced shifts in the mouse gut microbiome and metabolome increase susceptibility to Clostridium difficile infection. Nat Commun 2014; 5: 3114.

28.- Barra-Carrasco J, Paredes-Sabja D. Clostridium difficile spores: a major threat to the hospital environment. Future Microbiol 2014; 9: 475-86.

29.- Lawley T D, Walker A W. Intestinal colonization resistance. Immunology 2013; 138: 1-11.

30.- Walker A W, Lawley T D. Therapeutic modulation of intestinal dysbiosis. Pharmacol Res 2013; 69: 75-86.

31.- Higgins D, Dworkin J. Recent progress in Bacillus subtilis sporulation. FEMS Microbiol Rev 2012; 36: 131-48.

32.- Steiner E, Dago A E, Young D I, Heap J T, Minton N P, Hoch J A, et al. Multiple orphan histidine kinases interact directly with Spo0A to control the initiation of endospore formation in Clostridium acetobutylicum. Mol Microbiol 2011; 80: 641-54.

33.- Underwood S, Guan S, Vijayasubhash V, Baines S D, Graham L, Lewis R J, et al. Characterization of the sporulation initiation pathway of Clostridium difficile and its role in toxin production. J Bacteriol 2009; 191 : 7296-305.

34.- de Hoon M J, Eichenberger P, Vitkup D. Hierarchical evolution of the bacterial sporulation network. Curr Biol 2010; 20: R735-45.

35.- Fimlaid K A, Bond J P, Schutz K C, Putnam E E, Leung J M, Lawley T D, et al. Global analysis of the sporulation pathway of Clostridium difficile. PLoS Genet 2013; 9: e1003660.

36.- Janoir C, Deneve C, Bouttier S, Barbut F, Hoys $\mathrm{S}$, Caleechum L, et al. Insights into the adaptive strategies and pathogenesis of Clostridium difficile from in vivo transcriptomics. Infect Immun 2013; 81: 3757-69.

37.- Deakin L J, Clare S, Fagan R P, Dawson L F, Pickard D J, West M R, et al. The Clostridium difficile spo0A gene is a persistence and transmission factor. Infect Immun 2012; 80: 2704-11.

38.- Sunkesula V C, Kundrapu S, Jury L A, Deshpande A, Sethi A K, Donskey C J. Potential for transmission of spores by patients awaiting laboratory testing to confirm suspected Clostridium difficile infection. Infect Control Hosp Epidemiol 2013; 34: 306-8.

39.- Paredes-Sabja D, Sarker M R. Adherence of Clostridium difficile spores to Caco-2 cells in culture. J Med Microbiol 2012; 61: 1208-18. 
40.- Schlecht U, St Onge R P, Walther T, Francois J M, Davis R W. Cationic amphiphilic drugs are potent inhibitors of yeast sporulation. PLoS One 2012; 7: e42853.

41.- Louie T J, Miller M A, Mullane K M, Weiss K, Lentnek A, Golan Y, et al. Fidaxomicin versus vancomycin for Clostridium difficile infection. N Engl J Med 2011; 364: 422-31.

42.- Babakhani F, Bouillaut L, Gómez A, Sears P, Nguyen L, Sonenshein A L. Fidaxomicin inhibits spore production in Clostridium difficile. Clin Infect Dis 2012; 55 Suppl 2: S162-9.

43.- Paredes-Sabja D, Shen A, Sorg J A. Clostridium difficile spore biology: sporulation, germination and spore structural proteins. Trends Microbiol 2014; 22: 406-16

44.- Eyre D W, Cule M L, Wilson D J, Griffiths D, Vaughan A, O'Connor L, et al. Diverse sources of $C$. difficile infection identified on wholegenome sequencing. N Engl J Med 2013; 369: 1195-205.

45.- McFarland L V, Mulligan M E, Kwok R Y, Stamm W E. Nosocomial acquisition of Clostridium difficile infection. N Engl J Med 1989; 320: 204-10.

46.- Bobulsky G S, Al-Nassir W N, Riggs M M, Sethi A K, Donskey C J. Clostridium difficile skin contamination in patients with $C$. difficileassociated disease. Clin Infect Dis 2008; 46: 447-50.

47.- Landelle C, Verachten M, Legrand P, Girou E, Barbut F, Buisson C B. Contamination of healthcare workers' hands with Clostridium difficile spores after caring for patients with C. difficile infection. Infect Control Hosp Epidemiol 2014; 35: 10-15.

48.- Guerrero D M, Nerandzic M M, Jury L A, Jinno S, Chang S, Donskey C J. Acquisition of spores on gloved hands after contact with the skin of patients with Clostridium difficile infection and with environmental surfaces in their rooms. Am J Infect Control 2012; 40: 556-8.

49.- Shaughnessy M K, Micielli R L, DePestel D D, Arndt J, Strachan C L, Welch K B, et al. Evaluation of hospital room assignment and acquisition of Clostridium difficile infection. Infect Control Hosp Epidemiol 2011; 32: 201-6.

50.- Fekety R, Kim K H, Batts D H, Browne R A, Cudmore M A, Silva J, Jr., et al. Studies on the epidemiology of antibiotic-associated Clostridium difficile colitis. Am J Clin Nutr 1980; 33: 2527-32.

51.- Fekety R, Kim K H, Brown D, Batts D H, Cudmore M, Silva J Jr. Epidemiology of antibiotic-associated colitis; isolation of Clostridium difficile from the hospital environment. Am J Med 1981; 70: 906-8.

52.- Kim K H, Fekety R, Batts D H, Brown D, Cudmore M, Silva J, Jr., et al. Isolation of Clostridium difficile from the environment and contacts of patients with antibiotic-associated colitis. J Infect Dis 1981; 143: 42-50.

53.- Fawley W N, Wilcox M H. Molecular epidemiology of endemic Clostridium difficile infection. Epidemiol Infect 2001; 126: 343-50.

54.- Fawley W N, Freeman J, Wilcox M H. Evidence to support the existence of subgroups within the UK epidemic Clostridium difficile strain (PCR ribotype 1). J Hosp Infect 2003; 54: 74-7.

55.- Roberts K, Smith C F, Snelling A M, Kerr K G, Banfield K R, Sleigh P A, et al. Aerial dissemination of Clostridium difficile spores. BMC Infect Dis 2008; 8: 7.

56.- Setlow P. I will survive: DNA protection in bacterial spores. Trends Microbiol 2007; 15: 172-80.

57.- Cowan A E, Olivastro E M, Koppel D E, Loshon C A, Setlow B, Setlow P. Lipids in the inner membrane of dormant spores of Bacillus species are largely immobile. Proc Natl Acad Sci U S A 2004; 101: 7733-8.

58.- Paredes-Sabja D, Setlow P, Sarker M R. Germination of spores of Bacillales and Clostridiales species: mechanisms and proteins involved. Trends in Microbiol 2011; 19: 85-94.

59.- Warth AD, Strominger J L. Structure of the peptidoglycan of bacterial spores: occurrence of the lactam of muramic acid. Proc Natl Acad Sci U S A 1969; 64: 528-35.

60.- Warth A D, Strominger J L. Structure of the peptidoglycan from spores of Bacillus subtilis. Biochemistry 1972; 11: 1389-96.

61.- Makino S, Moriyama R. Hydrolysis of cortex peptidoglycan during bacterial spore germination. Med Sci Monit 2002; 8: RA119-27.

62.- Setlow P. Spores of Bacillus subtilis: their resistance to and killing by radiation, heat and chemicals. J Appl Microbiol 2006; 101: 514-25.

63.- Klobutcher L A, Ragkousi K, Setlow P. The Bacillus subtilis spore coat provides "eat resistance" during phagocytic predation by the protozoan Tetrahymena thermophila. Proc Natl Acad Sci USA 2006; 103: 165-70.

64.- Laaberki M H, Dworkin J. Role of spore coat proteins in the resistance of Bacillus subtilis spores to Caenorhabditis elegans predation. J Bacteriol 2008; 190: 6197-203.

65.- Barra-Carrasco J, Olguín-Araneda V, Plaza-Garrido A, Miranda-Cárdenas C, Cofré-Araneda G, Pizarro-Guajardo M, et al. The Clostridium difficile exosporium cysteine (CdeC)-rich protein is required for exosporium morphogenesis and coat assembly. J Bacteriol 2013; 195: 3863-75.

66.- Paredes-Sabja D, Sarker M R. Interactions between Clostridium perfringens spores and Raw 264.7 macrophages. Anaerobe 2012; 18 : 148-56.

67.- Permpoonpattana P, Phetcharaburanin J, Mikelsone A, Dembek M, Tan S, Brisson M C, et al. Functional characterization of Clostridium difficile spore coat proteins. J Bacteriol 2013; 195: 1492-503.

68.- Paredes-Sabja D, Sarker N, Setlow B, Setlow P, Sarker MR. Roles of DacB and Spm proteins in Clostridium perfringens spore resistance to moist heat, chemicals and UV radiation. Appl Environ Microbiol 2008; 74: 3730-8.

69.- Paredes-Sabja D, Setlow B, Setlow P, Sarker M R. Characterization of Clostridium perfringens spores that lack SpoVA proteins and dipicolinic acid. J Bacteriol 2008; 190: 4648-59.

70.- Deneve C, Janoir C, Poilane I, Fantinato C, Collignon A. New trends in Clostridium difficile virulence and pathogenesis. Int J Antimicrob Agents 2009; 33 Suppl 1: S24-S28.

71.- McFarland LV. Alternative treatments for Clostridium difficile disease: what really works? J Med Microbiol 2005; 54: 101-11.

72.- Rineh A, Kelso M J, Vatansever F, Tegos G P, Hamblin M R. Clostridium difficile infection: molecular pathogenesis and novel therapeutics. Expert Rev Anti Infect Ther 2014; 12: 131-50.

73.- Lipp M J, Pagovich O E, Rabin D, Min A D, Bernstein B B. Prevalence of diverticulosis in recurrent Clostridium difficile infection. World J Gastroenterol 2010; 16: 345-7.

74.- Dingle T C, Mulvey G L, Armstrong G D. Mutagenic analysis of the Clostridium difficile flagellar proteins, FliC and FliD, and their contribution to virulence in hamsters. Infect Immun 2011; 79: 4061-7.

75.- Drudy D, O’Donoghue D P, Baird A, Fenelon L, O'Farrelly C. Flow cytometric analysis of Clostridium difficile adherence to human intestinal epithelial cells. J Med Microbiol 2001; 50: 526-34.

76.- Britton R A, Young V B. Role of the intestinal microbiota in resistance to colonization by Clostridium difficile. Gastroenterology 2014; 146: 1547-53.

77.- Dawson L F, Valiente E, Donahue E H, Birchenough G, Wren B W. Hypervirulent Clostridium difficile PCR-ribotypes exhibit resistance to widely used disinfectants. PLoS One 2011; 6: e25754.

78.- Pérez J, Springthorpe V S, Sattar S A. Clospore: a liquid medium for producing high titers of semi-purified spores of Clostridium difficile. J AOAC Int 2011; 94: 618-26.

79.- Lawley T D, Clare S, Deakin L J, Goulding D, Yen J L, Raisen C, et al. Use of purified Clostridium difficile spores to facilitate evaluation of health care disinfection regimens. Appl Environ Microbiol 2010; 76: 6895-900.

80.- Wullt M, Odenholt I, Walder M. Activity of three disinfectants and acidified nitrite against Clostridium difficile spores. Infect Control Hosp Epidemiol 2003; 24: 765-8.

81.- Cowan R E, Manning A P, Ayliffe G A, Axon A T, Causton J S, Cripps N F, et al. 
Aldehyde disinfectants and health in endoscopy units. British Society of Gastroenterology Endoscopy Committee. Gut 1993; 34: 1641-5.

82.- Alfa M J, Lo E, Wald A, Dueck C, DeGagne P, Harding G K. Improved erradication of Clostridium difficile spores from toilets of hospitalized patients using an accelerated hydrogen peroxide as the cleaning agent. BMC Infect Dis 2010; 10: 268.

83.- Roberts K, Smith C F, Snelling A M, Kerr K G, Banfield K R, Sleigh P A, et al. Aerial dissemination of Clostridium difficile spores. BMC Infect Dis 2008; 8: 7.

84.- Baines S D, O'Connor R, Saxton K, Freeman J, Wilcox M H. Activity of vancomycin against epidemic Clostridium difficile strains in a human gut model. J Antimicrob Chemother 2009; 63: 520-5.

85.- Freeman J, Baines S D, Saxton K, Wilcox M H. Effect of metronidazole on growth and toxin production by epidemic Clostridium difficile PCR ribotypes 001 and 027 in a human gut model. J Antimicrob Chemother 2007; 60: 83-91.

86.- Chilton C H, Freeman J, Baines S D, Crowther G S, Nicholson S, Wilcox M H. Evaluation of the effect of oritavancin on Clostridium difficile spore germination, outgrowth and recovery. J Antimicrob Chemother 2013; 68: 2078-82.

87.- Freeman J, Marquis M, Crowther G S, Todhunter S L, Fawley W N, Chilton C H, et al. Oritavancin does not induce Clostridium difficile germination and toxin production in hamsters or a human gut model. J Antimicrob Chemother 2012; 67: 2919-26.

88.- Hernández-Rocha C, Naour S, Álvarez-Lobos M, Paredes-Sabja D. Infecciones causadas por Clostridium difficile: una visión actualizada. Rev Chilena Infectol 2012; 29: 434-45.

89.- Bouza E. Consequences of Clostridium difficile infection: understanding the healthcare burden. Clin Microbiol Infect 2012; 18 Suppl 6: 5-12.
90.- Babakhani F, Bouillaut L, Sears P, Sims C, Gómez A, Sonenshein A L. Fidaxomicin inhibits toxin production in Clostridium difficile. J Antimicrob Chemother 2013; 68: 515-22.

91.- Allen C A, Babakhani F, Sears P, Nguyen L, Sorg J A. Both fidaxomicin and vancomycin inhibit outgrowth of Clostridium difficile spores. Antimicrob Agents Chemother 2013; 57: 664-7.

92.- Francis M B, Allen C A, Shrestha R, Sorg J A. Bile acid recognition by the Clostridium difficile germinant receptor, $\mathrm{CspC}$, is important for establishing infection. PLoS Pathog 2013; 9: e1003356.

93.- Adams C M, Eckenroth B E, Putnam E E, Doublie S, Shen A. Structural and functional analysis of the $\mathrm{CspB}$ protease required for Clostridium spore germination. PLoS Pathog 2013; 9: e1003165.

94.- Sussman M D, Setlow P. Cloning, nucleotide sequence, and regulation of the Bacillus subtilis gpr gene, which codes for the protease that initiates degradation of small, acid-soluble proteins during spore germination. J Bacteriol 1991; 173: 291-300.

95.- Nerandzic M M, Donskey C J. Activate to eradicate: inhibition of Clostridium difficile spore outgrowth by the synergistic effects of osmotic activation and nisin. PLoS One 2013; 8: e54740.

96.- Escobar-Cortés K, Barra-Carrasco J, ParedesSabja D. Proteases and sonication specifically remove the exosporium layer of spores of Clostridium difficile strain 630. J Microbiol Methods 2013; 93: 25-31.

97.- Paredes-Sabja D, Sarker M R. Germination response of spores of the pathogenic bacterium Clostridium perfringens and Clostridium difficile to cultured human epithelial cells. Anaerobe 2011; 17: 78-84.

98.- Paredes-Sabja D, Torres J A, Setlow P, Sarker M R. Clostridium perfringens spore germination: characterization of germinants and their receptors. J Bacteriol 2008; 190: 1190-201. 99.- Nerandzic M M, Donskey C J. Triggering germination represents a novel strategy to enhance killing of Clostridium difficile spores. PLoS One 2010; 5: e12285.

100.-Sorg J A, Sonenshein A L. Bile salts and glycine as cogerminants for Clostridium difficile spores. J Bacteriol 2008; 190: 2505-12.

101.-Heeg D, Burns D A, Cartman S T, Minton N P. Spores of Clostridium difficile clinical isolates display a diverse germination response to bile salts. PloS one 2012; 7: e32381.

102.-Howerton A, Patra M, Abel-Santos E. A new strategy for the prevention of Clostridium difficile infection. J Infect Dis 2013; 207: 1498-504.

103.-Boyce J M, Havill N L, Moore B A. Terminal decontamination of patient rooms using an automated mobile UV light unit. Infect Control Hosp Epidemiol 2011; 32: 737-42.

104.-Saujet L, Monot M, Dupuy B, Soutourina O, Martin-Verstraete I. The key sigma factor of transition phase, $\mathrm{SigH}$, controls sporulation, metabolism, and virulence factor expression in Clostridium difficile. J Bacteriol 2011; 193 : 3186-96.

105.-Harry K H, Zhou R, Kroos L, Melville S B. Sporulation and enterotoxin (CPE) synthesis are controlled by the sporulation-specific sigma factors SigE and SigK in Clostridium perfringens. J Bacteriol 2009; 191: 2728-42.

106.-Pereira F C, Saujet L, Tome A R, Serrano M, Monot M, Couture-Tosi E, et al. The spore differentiation pathway in the enteric pathogen Clostridium difficile. PLoS Genet 2013; 9: e1003782.

107.-Underwood S, Guan S, Vijayasubhash V, Baines S D, Graham L, Lewis R J, et al. Characterization of the sporulation initiation pathway of Clostridium difficile and its role in toxin production. J Bacteriol 2009; 191 : 7296-305.

108.-Deng K, Plaza-Garrido A, Torres J A, Paredes-Sabja D. Survival of Clostridium difficile spores at low temperatures. Food Microbiol 2015; 46 (4): 218-21. 